\section{Un modelo de atención en salud al pueblo wayúu en la frontera colombo-venezolana}

\author{
María Beatriz Duarte Gómez, ${ }^{1}$ \\ Martha Liliana Ortega Alegría, \\ Luis Hernando Mora Ríos ${ }^{3}$ y \\ Noly Coromoto Fernández ${ }^{4}$
}

Forma de citar: Duarte Gómez MB, Ortega Alegría ML, Mora Ríos LH, Coromoto Fernández N. Un modelo de atención en salud al pueblo wayúu en la frontera colombo-venezolana. Rev Panam Salud Publica. 2011;30(3):272-8.

\section{SINOPSIS}

En reuniones y talleres con comunidades wayúu y funcionarios de gobierno de Colombia y Venezuela, tras validar el diagnóstico de salud y sus determinantes, se identificaron componentes para un modelo de atención binacional en salud destinado al pueblo wayúu que habita la frontera entre ambos países. Si bien ha habido avances en la atención sanitaria intercultural en ambos países, todavía hace falta fortalecer algunos aspectos de la legislación y la organización de los servicios para hacer posible la garantía del derecho a la salud. Se debe asegurar la asignación de nuevos recursos nacionales - o la redistribución de los actuales-y la gestión de recursos internacionales para ejecutar un proyecto piloto en el corto plazo e implementar el modelo en el mediano plazo.

Palabras clave: Salud indígena; salud fronteriza; comunidades vulnerables; sistemas de salud; cobertura de los servicios de salud; Colombia; Venezuela.

\footnotetext{
Instituto Nacional de Salud Pública, Cuernavaca, Morelos, México. La correspondencia se debe dirigir a María Beatriz Duarte Gómez, mduarte@insp.mx

2 Universidad del Cauca, Facultad de Ciencias de la Salud, Popayán, Colombia.

3 Universidad de Antioquia, Colombia.

4 Ministerio del Poder Popular para la Salud, Caracas, Venezuela.
}

En 2004, con el propósito de fortalecer el trabajo sanitario conjunto en la frontera entre Colombia y Venezuela, se creó el Comité Técnico Binacional en Salud de la Comisión Presidencial de Integración y Asuntos Fronterizos Colombo-Venezolanos (COPIAF). ${ }^{5}$ Este comité planteó, entre otras estrategias, desarrollar un modelo de atención intercultural para el pueblo wayúu, teniendo en cuenta que es histórica y culturalmente uno solo, que su territorio ancestral es el mismo y que algunos de los problemas y determinantes de salud son similares en ambos lados de la frontera (1). La mayoría de los wayúu tienen las dos nacionalidades - colombiana y venezolana - y hay una gran movilidad fronteriza tanto comercial como familiar.

El presente diagnóstico de la situación de salud se llevó a cabo usando el enfoque de determinantes sociales de la Organización Mundial de la Salud (OMS), que los define como las condiciones en que las personas nacen, crecen, viven, trabajan y envejecen, así como los ejes de la promoción de la salud propuestos en la Carta de Ottawa en 1986, y las políticas y convenios internacionales sobre la salud de los pueblos indígenas que promueven la transformación de los sistemas sanitarios y el desarrollo de modelos alternativos de atención (3-8). Su objetivo fue identificar elementos que contribuyan a establecer el modelo de atención en salud para el pueblo wayúu en el cual viene trabajando la COPIAF.

\section{EL CONTEXTO}

En las últimas décadas en las Américas se han venido reivindicando los derechos de los pueblos indígenas, quienes durante más de 500 años han sido irrespetados, incomprendidos y sojuzgados y con quienes el mundo occidental tiene una deuda impagable. La lucha de los pueblos por sus derechos y la posición de algunos sectores de la sociedad respecto a compensar el daño han resultado en convenios y declaraciones internacionales y en movimientos de apoyo de la sociedad civil a sus demandas de autonomía y respeto (8-10). Recientemente, el ORAS ha formalizado una política de interculturalidad a través de la Comisión Andina de Salud, espacio donde se definen lineamientos para las políticas de salud de los pueblos indígenas y los países se comprometen a adecuar sus políticas y programas $(11,12)$.

Por otro lado, las políticas internacionales, como la Iniciativa Salud de los Pueblos Indígenas de la Organización Panamericana de la Salud (OPS) y la Carta

El modelo de COPIAF también forma parte del plan de salud intercultural del Organismo Andino de Salud (ORAS) (2). 
de Ottawa ${ }^{6}$ proponen intervenciones sobre los determinantes sociales de la salud y el bienestar y reconocen la cultura y la diversidad cultural como factores decisivos en el cuidado de la salud $(6,9)$.

Algunas características del cuidado de la salud en la cultura wayúu se relacionan con el tipo de enfermedad. Así las ayulee (naturales), consideradas benignas, generalmente se tratan en el ámbito doméstico por parte de las abuelas o las madres, sobre todo con plantas. Las wanülü̈ü, en cambio, se caracterizan por síntomas "espectaculares" —como hematemesis o dolores internos- y requieren la intervención de terapeutas tradicionales (piaachi), quienes tienen procedimientos diagnósticos y terapéuticos propios de su cultura (13).

Una parte importante del contexto es el sistema político y el sistema de salud de cada país. En este caso, Colombia y Venezuela tienen sistemas sanitarios con objetivos similares (equidad y universalidad) y bases constitucionales parecidas (salud como derecho y reconocimiento de la pluri-etnicidad) pero difieren en su organización y sus bases ideológicas y políticas. Colombia apunta a garantizar la atención de la salud a través del aseguramiento universal con paquetes de servicios, con amplia participación del sector privado en un mercado más o menos regulado y con un papel mínimo del Estado en la administración de recursos y en la prestación de estos servicios (14-19). A pesar de los avances en términos de instrumentos legales que favorecen la adaptación del sistema a las características de las comunidades indígenas y la apertura de espacios para su participación, ${ }^{7}$ la forma en que funciona deja a personas sin servicios, aun estando aseguradas, principalmente en comunidades dispersas (20-23).

En este sentido, se han abierto espacios para que las organizaciones indígenas actúen, aunque con muchas dificultades, como empresas aseguradoras y prestadoras de servicios de salud, y efectúen adaptaciones culturales (24). Ha habido también experiencias locales como la del Hospital de Nazareth, en la Alta Guajira, donde funciona un modelo de atención intercultural desde hace más de tres décadas, que fue una iniciativa de su director, no de una política estatal. En este hospital casi la totalidad del personal es indígena (incluyendo algunos médicos), existen adaptaciones locativas para que el paciente y su familia estén en un ambiente físico similar al de su hogar y se facilita la relación con los agentes de medicina tradicional. Las enormes dificultades para la comunicación y referencia a hospitales de mayor complejidad en Colombia han producido una relación estrecha con el Hospital de Paraguaipoa en Venezuela, tanto en actividades preventivas (p. ej. vacunación) como de referencia de pacientes.

\footnotetext{
6 En este documento las condiciones y requisitos para la salud incluyen "paz, educación, vivienda, alimentación, renta, ecosistema estable, justicia social y equidad" (6).

7 Ariza, JF. Insumos para la formulación del modelo de salud para la población wayúu en la zona fronteriza colombo-venezolana. Informe final del contrato de servicios con la Organización Panamericana de la Salud. Bogotá, Colombia; 2008. (Documento no
}

Venezuela por su parte tiene un sistema de salud que procura la cobertura universal para los no asegurados a través de la red pública, el papel del Estado es preponderante y la ejecución de las políticas de interculturalidad tiene un avance muy importante como política pública. Destacan la Ley Orgánica de Pueblos y Comunidades Indígenas y la creación de una Dirección de Salud Indígena en el Ministerio del Poder Popular para la Salud y Protección Social, que han dado lugar a iniciativas como el Sistema de Atención y Orientación al indígena (SAOI), los "facilitadores interculturales" y las "defensoras de la salud". Hay además otras propuestas, como la odontología transcultural de la Universidad del Zulia $(25,26)$.

\section{EL ESTUDIO}

El presente trabajo se dividió en dos etapas. En la primera, conjuntamente con las comunidades y las instituciones, se validaron los diagnósticos de salud y sus determinantes obtenidos de estadísticas y estudios previamente realizados por diferentes organismos $(3,4,19,20)$. La validación se realizó a través de talleres, utilizándose una guía para identificar los principales problemas de salud y sus determinantes, la mejor manera de ofrecer servicios e información al pueblo wayúu, sugerencias para garantizar el éxito de un programa binacional y los aportes que ambos países podrían hacer (cuadro 1). Con base en esta información se inició la segunda etapa, consistente en preparar la propuesta con los lineamientos para el modelo de atención y su validación y enriquecimiento en sendas reuniones interinstitucionales y comunitarias. Adicionalmente, se efectuaron actividades de observación en comunidades urbanas y rurales y en unidades de salud de ambos lados de la frontera, así como entrevistas con informantes clave del sector salud en los niveles nacional, regional y local.

\section{RESULTADOS}

\section{Diagnóstico de salud de la población wayúu}

Según el censo de 2001, en Venezuela había 285446 wayúu en el estado del Zulia y, en Colombia, 149827 en el departamento de la Guajira $(27,28)$. Al no contar con un sistema de información que permitiera analizar la variable de pertenencia étnica de las personas atendidas y estimar con mayor exactitud la magnitud de los problemas de salud de la población indígena, no fue posible realizar un diagnóstico de salud preciso. Sin embargo, en ambos países ya hay normas y proyectos piloto para la inclusión de esta variable en el sistema de información y se viene trabajando en el diagnóstico de salud con la metodología ASIS (análisis de la situación de salud) $(2,3)$. Se halló que las enfermedades carenciales y de la pobreza son las que caracterizan el perfil epidemiológico, con algunas particularidades relacionadas principalmente con la geografía (en especial zona desértica con escasez de agua) y con la cultura (p. ej. organización social y actividad económica). Las patologías más frecuentes incluyeron i) des- 
CUADRO 1. Reuniones con comunidades y funcionarios de gobierno de Colombia y Venezuela para identificar componentes de un modelo de atención en salud destinado al pueblo wayúu que habita la frontera entre ambos países

\begin{tabular}{llcllll}
\hline \multirow{2}{*}{$\begin{array}{c}\text { Tipo de } \\
\text { reunión (No.) }\end{array}$} & Lugar & $\begin{array}{c}\text { Asistentes } \\
\text { (No.) }\end{array}$ & & Lugar & $\begin{array}{c}\text { Asistentes } \\
\text { (No.) }\end{array}$ & Observaciones \\
\cline { 2 - 4 } Institucional (2) & Páez & 20 & Riohacha & 15 & $\begin{array}{l}\text { En ambas hubo indígenas como representantes de } \\
\text { instituciones de salud. }\end{array}$ \\
Comunitaria (1) & Páez & $100^{\mathrm{a}}$ & & $\begin{array}{c}\text { Flor del } \\
\text { Paraíso }\end{array}$ & 60 & $\begin{array}{l}\text { A la reunión de Páez asistieron vacunadoras, promotoras, } \\
\text { facilitadoras y otros líderes comunitarios. En Flor del } \\
\text { Paraíso se aprovechó una asamblea de esa comunidad } \\
\text { citada para otro asunto de salud. }\end{array}$ \\
\hline
\end{tabular}

Fuente: elaborado por los autores.

a Se trató de representantes de 30 comunidades distintas.

nutrición, infecciones respiratorias y gastrointestinales en menores de cinco años de edad, ii) infecciones de transmisión sexual, cáncer cérvico-uterino, hipertensión arterial y las derivadas de la violencia en personas adultas y iii) caries y otros problemas dentales en todas las edades $(29,30)$.

Los principales determinantes de la salud identificados fueron falta de agua potable, escasez de alimentos, pobreza, acceso limitado y fragmentado a servicios de salud. También lo fueron la falta de educación intercultural y los cambios culturales que, según las personas entrevistadas y algunos autores, ha sufrido el pueblo wayúu a raíz de la migración, de la presencia de grupos armados, de la explotación minera y de los programas asistenciales del Estado (31).

Asimismo, durante las visitas a las comunidades se constataron las diferencias de acceso a los servicios de salud, encontrándose que en el lado colombiano existen mayores obstáculos geográficos (p. ej. falta de carreteras y de transporte) y funcionales (p. ej. trámites y fragmentación de servicios) y que la supervisión por parte del Estado es deficiente.

\section{ELEMENTOS PARA EL MODELO}

La propuesta fue construida colectivamente, considerando los componentes necesarios en cualquier modelo de atención de salud, pero con algunas particularidades de relevancia en zonas indígenas. El modelo debe tener en cuenta de manera central la relación de la salud del indígena con lo espiritual, con la familia, con el medio ambiente, y con la armonía de la persona consigo misma y con los demás. Tal como lo recomienda el ORAS, la interculturalidad, la integralidad y el respeto de los derechos fundamentales deben ser transversales a toda política de salud indígena (32). A continuación se reseñan los elementos mínimos propuestos (figura 1).

FIGURA 1. Elementos de un modelo binacional de salud para la población wayúu en la frontera Colombia-Venezuela

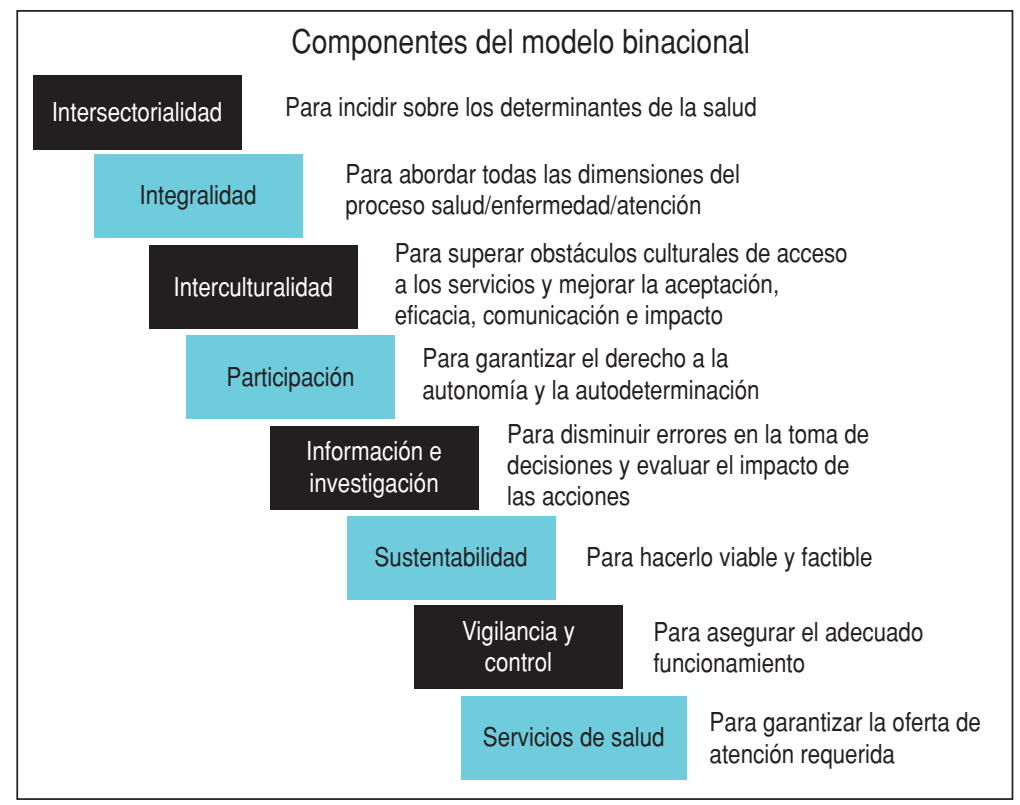

Fuente: elaborado por los autores. 


\section{Intersectorialidad}

En mayor o menor medida, la salud debe formar parte de todas las políticas públicas, teniendo como centro el desarrollo humano integral $(33,34)$. Tal medida significa que quienes toman decisiones en sectores como agua, seguridad alimentaria, infraestructura, trabajo, educación, comunicaciones, vivienda, presupuesto, seguridad territorial y medio ambiente tienen que ser conscientes de que sus acciones $u$ omisiones conllevan un impacto sobre la salud. La Comisión Binacional de Alto Nivel Colombia-Venezuela y el COPIAF podrían ser los espacios idóneos para lograr estos compromisos políticos.

\section{Integralidad}

La aplicación del principio de integralidad en este modelo de atención consistiría fundamentalmente en brindar servicios de salud teniendo en cuenta los aspectos bio-psico-sociales y espirituales del proceso salud-enfermedad-atención. Debería contar con equipos multidisciplinarios; enfocar las acciones no solo en el individuo sino también en su familia; evitar la fragmentación de la atención médica; complementar la medicina occidental con la tradicional; garantizar acciones de promoción, prevención y atención; asegurar el acceso a todos los niveles de atención e incluir el cuidado sanitario de los animales domésticos, por solicitud expresa de las comunidades.

\section{Interculturalidad}

La interculturalidad ha sido definida como "un proceso social interactivo de reconocimiento y respeto de las diferencias existentes en una o varias culturas . . . indispensable para construir una sociedad justa ..." y en salud implica "la capacidad de actuar equilibradamente entre conocimientos, creencias y prácticas culturales diferentes, respecto a la salud y a la enfermedad ...", lo cual implica un proceso de diálogo entre los sistemas médicos tradicional y occidental y entre estos y la comunidad en un ambiente de confianza, respeto y autodeterminación (35-38). Sin embargo, el modelo médico hegemónico biomédico y la posición social subordinada de los indígenas han dificultado esta relación horizontal, dando lugar a conflictos entre los agentes de las dos medicinas $(39,40)$. Es preciso desarrollar recursos humanos competentes para la atención intercultural y adecuar los servicios de salud a las concepciones y prácticas de la comunidad tales como lenguaje, infraestructura, dieta, horarios, albergue, así como señalizar los servicios en las instituciones en lengua wayúu y garantizar la atención materna.

El consenso en los talleres fue la necesidad de trabajar las dos medicinas en forma articulada pero conservando su independencia, teniendo en cuenta que puede haber una complementariedad por iniciativa del paciente o del terapeuta (38). Se propuso garantizar la disponibilidad de plantas medicinales en los hospitales y, si bien la comunidad manifestó que quiere mejorar su conocimiento sobre la medicina occidental, prefiere hacerlo a través de personal nativo capacitado.

\section{Participación social}

La participación social, entendida como la posibilidad de acceso real de la población al control de los procesos que afectan su salud, está enfocada a garantizar el derecho a la autonomía y a la autodeterminación de los pueblos. En las consultas del presente estudio se propuso generar instancias y mecanismos de participación en las decisiones, que sean acordes con las formas organizativas de los wayúu y la formación de comités municipales que supervisen el cumplimiento de los acuerdos.

\section{Información e investigación}

Contar con información veraz, confiable y oportuna es indispensable para la toma de decisiones adecuadas. Se requiere un sistema de información que incluya la variable etnia y el análisis epidemiológico con enfoque intercultural, acordándose mecanismos para compartir información entre los dos países. En los casos de problemas de salud sobre los que no existe el conocimiento suficiente que permita el planteamiento de intervenciones, será relevante llevar a cabo actividades binacionales de investigación dirigidas precisamente a aprender acerca de esos problemas a medida que se vayan planteando.

\section{Sustentabilidad}

Para que el modelo pueda operar a largo plazo es necesario asegurar el compromiso político de todos los niveles de gobierno y la comunidad, como requisito para que los cambios de autoridades no signifiquen un freno a la vigencia de los convenios previamente suscritos, a la expedición de normas y al empoderamiento de las comunidades. Se debe garantizar la asignación de nuevos recursos nacionales - o la redistribución de los actuales- y la gestión de recursos internacionales para ejecutar el proyecto piloto en el corto plazo e implementar el modelo en el mediano plazo.

\section{Vigilancia y control}

Se observaron deficiencias en la vigilancia y control de los servicios que deberían prestarse en la comunidad fronteriza del estudio, principalmente en Colombia. Tanto la comunidad como los organismos de control deben tener información clara sobre los derechos, la legislación, los presupuestos asignados y los demás insumos necesarios para que la veeduría social a los servicios pueda efectuarse.

\section{Servicios de salud}

Los servicios de salud son un componente clave del modelo porque constituyen lo mínimo que el Es- 
tado debe garantizar a la comunidad wayúu. Estos servicios deberán ser oportunos — disponibles siempre que sean requeridos- y accesibles desde los puntos de vista geográfico, económico, funcional y cultural. Asimismo, deberán tener un enfoque integral, que incluya todos los niveles de atención y los programas pertinentes según el perfil epidemiológico, el ciclo de vida completo, todas las dimensiones del ser humano y todas las enfermedades -las "occidentales" y las propias de la cultura wayúu.

\section{CONCLUSIONES}

Con base en los resultados obtenidos durante la realización del presente trabajo, se pueden extraer las siguientes conclusiones, mientras que en el cuadro 2 se explicitan recomendaciones dirigidas a cada uno de los dos países en cuyas fronteras habita la comunidad wayúu.

- El esfuerzo de la COPIAF debe ir acompañado de una mayor participación comunitaria en la búsqueda de estrategias que se adapten a la organización social del pueblo wayúu.

- Si se ha de garantizar la interculturalidad, el modelo debe basarse en personal nativo capacitado en todos los niveles de atención del sistema de salud.

- El abordaje de los problemas de salud y la acción sobre sus determinantes sociales requieren del lide- razgo del sector salud para mejorar el desarrollo social y humano dentro del respeto por la autonomía de la comunidad.

- Aun con los avances conseguidos en interculturalidad, cobertura sanitaria y normativa tanto en Colombia como en Venezuela, es necesario trabajar algunos aspectos de legislación y organización de sus servicios de salud para hacer posible la garantía del derecho a la salud.

- La interculturalidad y la integralidad son los principios básicos de un modelo de atención para población indígena.

- Es preciso crear un grupo de trabajo binacional que diseñe, desarrolle y evalúe la prueba piloto del modelo de atención en la zona comprendida entre el Hospital Nazareth y el de Paraguaipoa.

- Es perentorio fortalecer la formación en salud de recursos humanos nativos en todos los niveles —auxiliar, técnico y profesional.

Agradecimientos. El presente trabajo fue posible gracias al financiamiento de EUROSOCIAL, al apoyo del Ministerio de Protección Social de Colombia, del Ministerio del Poder Popular para la Salud y Protección Social de Venezuela y del Instituto Nacional de Salud Pública de México; al esfuerzo de los equipos regionales y locales de salud de la Guajira y de Páez, y a la valiosa experiencia aportada por los líderes comunitarios de ambos países.

CUADRO 2. Recomendaciones para el desarrollo de un modelo binacional, intercultural e intersectorial dirigido al pueblo wayúu en la frontera Colombia-Venezuela

\begin{tabular}{|c|c|}
\hline A Colombia & A Venezuela \\
\hline $\begin{array}{l}\text { 1. Diseñar mecanismos efectivos para hacer cumplir normas vigentes } \\
\text { sobre concertación con las comunidades e información veraz y } \\
\text { completa sobre los servicios. } \\
\text { 2. Adaptación de algunas normas: } \\
\text { - Regular el mercadeo de las aseguradoras en comunidades } \\
\text { dispersas para garantizar integralidad y optimizar los recursos, } \\
\text { incluyendo cláusulas que eviten la fragmentación de contratos } \\
\text { (p. ej. en atención médica, laboratorio, farmacia y odontología). } \\
\text { - Garantizar en los contratos con los prestadores de servicios las } \\
\text { visitas médicas periódicas a las comunidades que disten } 1 \text { hora } \\
\text { o más de la unidad de salud en el medio de transporte habitual. } \\
\text { - Asegurar que haya personal auxiliar nativo bilingüe permanente } \\
\text { en las comunidades alejadas y dispersas, con remuneración y } \\
\text { facilidades de comunicación. } \\
\text { - Garantizar que la variable étnica se registre y se analice en el } \\
\text { sistema de información. } \\
\text { 3. Fortalecer la dependencia encargada de la salud de los indígenas } \\
\text { en el Ministerio de Protección Social y el apoyo al personal } \\
\text { departamental de la Guajira encargado de los asuntos de salud de } \\
\text { la frontera con Venezuela. } \\
\text { 4. Diseñar con las comunidades los mecanismos de participación } \\
\text { comunitaria y control social que garanticen autodeterminación, } \\
\text { autonomía y cumplimiento de las normas. } \\
\text { 5. Sistematizar, publicar y difundir información sobre culturas } \\
\text { indígenas que facilite la capacitación del personal de salud local y } \\
\text { la adecuación cultural de los servicios, y mejorar la investigación } \\
\text { sobre cultura médica tradicional. } \\
\text { 6. Facilitar el intercambio de experiencias entre prestadores y } \\
\text { aseguradoras indígenas de diferentes zonas del país y sus } \\
\text { respectivas comunidades. }\end{array}$ & $\begin{array}{l}\text { 1. Identificar los costos de la atención a los indígenas provenientes } \\
\text { de Colombia y clasificarlos por patologías y nivel de atención. } \\
\text { 2. Desarrollar un sistema único de información en salud. } \\
\text { 3. Fortalecer las acciones de vigilancia epidemiológica de salud } \\
\text { pública en coordinación con Colombia. } \\
\text { 4. Articular las diferentes modalidades de atención (p. ej. barrio } \\
\text { adentro, sistema tradicional, sistema regional) para optimizar los } \\
\text { recursos y ampliar la red de servicios. } \\
\text { 5. Garantizar la disponibilidad de medicamentos y la racionalidad en } \\
\text { su distribución según el perfil epidemiológico local. } \\
\text { 6. Mejorar la supervisión en el uso de protocolos y guías que } \\
\text { garanticen la calidad técnica de la atención. } \\
\text { 7. Sistematizar las experiencias de adaptación intercultural de las } \\
\text { políticas de salud a pueblos indígenas para orientar la } \\
\text { consolidación de los logros y los cambios necesarios. }\end{array}$ \\
\hline
\end{tabular}

Fuente: elaborado por los autores. 


\section{SYNOPSIS}

\section{A health care model for the Wayúu people on the Colombian-Venezuelan border}

At meetings and workshops with Wayúu communities and government officials from Colombia and Venezuela, after validating the analysis of the health situation and its determinants, the components of a binational health care model for the Wayúu people living on the border between the two countries were identified. Although both countries have made progress in intercultural health care, some aspects of their legislation and service organization still need to be strengthened to make the guaranteed right to health a real$i t y$. The allocation of new national resources-or the redistribution of current resources - should be ensured, as well as the management of international resources for conducting a short-term pilot project and implementing the model in the medium term.

Key words: indigenous health; border health; vulnerable groups; health systems; health services coverage; Colombia; Venezuela.

\section{REFERENCIAS}

1. Ministerio de la Protección Social, Colombia; Ministerio del Poder Popular para la Salud, Venezuela. Comité técnico binacional en salud de la Comisión Presidencial de Interacción y Asuntos Fronterizos colombo-venezolanos (COPIAF). Resumen ejecutivo; julio 2007.

2. Dirección Nacional de Salud Indígena. Comisión Andina de Salud Intercultural: en marcha incorporación de interculturalidad en salud. Revista Voces indígenas. 2007;2:42.

3. Ministerio de la Protección Social, Colombia; Ministerio del Poder Popular para la Salud, Venezuela. Propuesta de análisis de situación en salud en las fronteras colombo-venezolanas -ASISBogotá; septiembre 2006.

4. República de Colombia, República Bolivariana de Venezuela, Organización Panamericana de la Salud. Tratado de cooperación técnico científico para el fortalecimiento de la vigilancia y el control de las enfermedades de interés en salud pública y el diseño del modelo de atención en salud de la frontera colombo-venezolana. Marzo; 2007. (Documento fotocopiado).

5. Solar O, Irwin A. A Conceptual Framework for Action on the Social Determinants of Health. Discussion paper for the Commission on Social Determinants of Health. Geneva: World Health Organization; 2007.

6. Primera Conferencia Internacional sobre la Promoción de la Salud, Canadá. Carta de Ottawa para la Promoción de la Salud; 1986. Disponible en: http:// www.paho.org/Spanish/hpp/ottawa charterSp.pdf Acceso el 7 de junio de 2011.

7. Organización de Naciones Unidas. Declaración de las Naciones Unidas sobre los derechos de los pueblos indígenas; 2007. Disponible en: http://www.un. org/esa/socdev/unpfii/es/drip.html Acceso el 7 de junio de 2011.

8. Organización Internacional del Trabajo. Convenio No.169 sobre pueblos indígenas y tribales en países independientes;
1989. Disponible en: http://www.ilo. org/public/spanish/region/ampro/ lima/publ/conv-169/convenio.shtml Acceso el 7 de junio de 2011.

9. Organización Panamericana de la Salud. Reunión de trabajo sobre pueblos indígenas y salud, Resolución V Salud de los pueblos indígenas. Winnipeg, Canadá; abril 1993. Disponible en: http://www.amro.who.int/span ish/ad/ths/os/indig-Resol-V.htm Acceso el 7 de junio de 2011.

10. Organización Panamericana de la Salud. Iniciativa Salud de los Pueblos Indígenas. Lineamientos estratégicos y plan de acción, 2003-2007. Disponible en: http:// www.üdh.ed.cr/comunidades/diversi dades/docs/div_enlinea/etnicidad, $\% 20$ plan $\% 20$ de $\% 20$ accion $\% 20-\% 20$ original. $\mathrm{htm}$ Acceso el 7 de junio de 2011.

11. Huertas B. Normatividad sobre pueblos indígenas en aislamiento, contacto reciente y contacto inicial en la Región Andina; 2010. Disponible en: http:// oras conhu.org/documentos/SI\%202\%20 Base $\% 20$ de $\% 20$ datos $\% 20$ Normas $\% 20$ PI AV\%20y\%20CI\%20Beatriz\%20Huer tas.pdf Acceso el 7 de junio de 2011.

12. Organismo Andino de Salud. Plan de trabajo, 2010. Disponible en: http:// orasconhu.org/index.php?IDIOMA= SP\&plantilla $=$ contenido\&ncategoria $1=$ 318 Acceso el 7 de junio de 2011.

13. Perrin M, Uliyuu-Machado J. El arte guajiro de curar frente a la medicina occidental. Boletín Indigenista Venezolano. 1980;19(16):39-200.

14. República de Colombia. Ley 100, 1993. Disponible en: http://www.colombia. com/actualidad/images/2008/leyes/ ley100.pdf Acceso el 7 de junio de 2011.

15. Cardona A. Participación de las entidades promotoras de salud (EPS) en el mercado del aseguramiento de salud, Colombia, 1995-1998. Rev Fac Nac Salud Publica. 1999;17(1):52-62.

16. Departamento Nacional de Planeación (DNP), Colombia. Consejo Nacional de Política Económica y Social (CONPES). Salud y seguridad social (documento
No. 2756). Santa Fe de Bogotá, Colombia: DNP; 1995.

17. Céspedes JE, Jaramillo I, Martínez R, Olaya S, Reynales J, Uribe C, et al. Efectos de la reforma de la seguridad social en salud en Colombia sobre la equidad en el acceso y la utilización de servicios de salud. Rev Salud Publica (Bogotá). 2000;2(2):145-64

18. Londoño JL, Frenk J. Pluralismo estructurado: hacia un modelo innovador para la reforma de los sistemas de salud en América Latina. Banco Interamericano de Desarrollo [documento de trabajo No. 353, 1997]. Disponible en: http://www.iadb.org/res/publica tions/pubfiles/pubWP-353.pdf Acceso el 7 de junio de 2011.

19. Ministerio de la Protección Social (MPS), Colombia. Primer informe nacional de calidad en la atención en salud “INCAS Colombia 2009". Bogotá: MPS; 2009.

20. Ministerio de la Protección Social (MPS), Colombia; Organización Panamericana de la Salud. Insumos para la conceptualización y discusión de una política de protección social en salud para los grupos étnicos de Colombia. Bogotá, Colombia: MPS/OPS; 2004.

21. Ministerio de la Protección Social (MPS), Colombia. Plan Nacional de Salud Pública, 2007-2010. Bogotá, Colombia: MPS; 2008.

22. Ministerio de la Protección Social, Colombia. Acciones adelantadas con grupos étnicos. Presentación en la Comisión Andina de Salud Intercultural; 2008.

23. Ruiz-Rodríguez M, De Oliveira $\mathrm{O}$, López S, Angulo ML, Salas-Mora M, Duarte-Gómez MB. El seguro de salud para población joven desplazada por el conflicto armado en Colombia: proceso de afiliación y garantía de derechos. Ponencia presentada en el XIX Congreso de la Asociación Latina de Sistemas de Salud (Bruselas, 11-13 de septiembre de 2008).

24. Organización Gonawindúa Tayrona. Salud Indígena en la Sierra Nevada de 
Santa Marta: ley de Se, Seyn Zare, Shenbuta. Bogotá, Colombia: Sirga; 2009.

25. República Bolivariana de Venezuela. Ley Orgánica de Pueblos y Comunidades Indígenas. Gaceta oficial No. 38.344 (27 de diciembre de 2005).

26. Pirona M, García N, Rincón MC. Odontología transcultural: Eje transversal del currículo de la Facultad de Odontología de la Universidad del Zulia (Parte I). Ciencia Odontologica. 2006:3(2):78-84

27. Instituto Nacional de Estadística, República Bolivariana de Venezuela. Censo de población y vivienda, 2001.

28. Departamento Nacional de Planeación, Colombia. Grupos étnicos. Cap. 8. En: Los pueblos indígenas de Colombia en el umbral del nuevo milenio. Población, cultura y territorio: bases para el fortalecimiento social y económico de los pueblos indígenas. Bogotá; 2004. Disponible en: http://www.dnp.gov.co/ PortalWeb/Programas / Desarrollo Territorial/OrdenamientoyDesarrol loTerritorial/Grupos\%c3\%89tnicos. aspx Acceso el 7 de junio de 2011.

29. Dirección General de Promoción Social, Ministerio de la Protección Social (MPS), Colombia. Diagnóstico y caracterización de la población indígena del departamento de la Guajira. Bogotá, Colombia: MPS; 2008.

30. Carruyo M. Análisis de la situación de salud de fronteras Páez-Venezuela, 2005-2006. Informe técnico de epidemiología municipal de salud. Epidemiología Regional de Salud del estado Zulia. Sistema Regional de Salud. Cara- cas, Venezuela: Ministerio del Poder Popular para la Salud; 2010.

31. Paz-Reverol C, Leal-Jerez M, AlarcónPuentes J, Valbuena-Chirinos C, GarcíaGavidia N, Flores- Díaz D. Ser curador wayúu en la globalización y no morir en el intento. Index Enferm (edición digital) 2010; 19(2-3). Disponible (con suscripción) en: http://www.indexf.com/index-enfermeria/v19n2-3/ 7270.php Acceso el 10 de febrero de 2011.

32. Huertas-Castillo B. Propuesta de sensibilización para la protección de los pueblos en aislamiento, contacto reciente y contacto inicial. Organismo Andino de Salud, 2009. Disponible en: http://orasconhu.org/documentos/SI $\% 205 \% 20 \% 20$ Prop $\% 20$ Mod $\% 20$ sen sib\%20y\%20cap\%20PAV\%20junio\%200 9.pdf Acceso el 9 de junio de 2011.

33. Organización Panamericana de la Salud. Reunión Subregional de Gerentes del Cono Sur y Área Andina, 2009. Disponible en: http:/ / www.paho org/English/D/D_NewsletterI0409. asp Acceso el 9 de junio de 2011.

34. Martinez NM. Las estrategias de integración en salud de América Latina ante la crisis económica global, para una mejor calidad de vida de nuestros pobladores y el cumplimiento de los Objetivos del Milenio. Disponible en: http:/ / www.alames.org/documen tos/EJE02-52.pdf Acceso el 9 de junio de 2011.

35. Organización Panamericana de la Salud. Una visión de salud intercultural para los pueblos indígenas de las Américas. Washington, D.C: OPS; 2008.

36. Cevallos R, Amores A. Prestación de servicios de salud en zonas con pueblos indígenas. Disponible en: http://new. paho.org/hq/dmdocuments/2009/ser vicios\%20salud \%20zonas\%20indige nas.pdf Acceso el 9 de junio de 2011

37. Aula intercultural. Interculturalidad. Disponible en: http://www.aulainter cultural.org/article.php3?id_article $=54$ Acceso el 9 de junio de 2011.

38. Huertas-Castillo B. Propuesta de Reglamento para el personal de los diferentes sectores responsables de brindar atención integral a poblaciones en contacto reciente y contacto inicial. Organismo Andino de Salud. Disponible en: http://orasconhu.org/documentos/SI \%204\%20Prop\%20Reglamento\%20para \%20el\%20personal\%20PAV.pdf Acceso el 9 de junio de 2011.

39. Menéndez E. Antropología Médica: Desigualdades y transacciones. Cuadernos de la Casa Chata. CIESAS. 1990;179:121.

40. Duarte-Gómez MB. Medicina occidental y otras alternativas: es posible su complementariedad? Reflexiones conceptuales. Cad Saude Publica. 2003;19 (2):635-43.

Manuscrito recibido el 25 de febrero de 2010. Aceptado para publicación, tras revisión, el 5 de mayo de 2011. 